\title{
Does a Free-Trial Approach Increase Purchase and Use of a Household Water Treatment and Safe Storage Product in Rural Haiti?
}

\author{
Feng-Jen Tsai, ${ }^{1,2 *}$ Michael Wu, ${ }^{3}$ and Chia-Ping Lin ${ }^{1}$ \\ ${ }^{1}$ Master Program in Global Health and Development, College of Public Health, Taipei Medical University, Taipei City, Taiwan; ${ }^{2}$ Ph.D. Program in \\ Global Health and Health Security, College of Public Health, Taipei Medical University, Taipei City, Taiwan; ${ }^{3}$ Centers for Disease Control and
}

Prevention, Atlanta, Georgia

\begin{abstract}
A cluster, randomized control trial was conducted to assess the effects of social marketing approach on purchase rates and water treatment behavior of Klorfasil, a chlorine-based household water treatment product among seven villages in Thomassique, Haiti, from May to December 2016. Villages were randomized to the free-trial (257 households) or cost-sharing (240 households) group. Households in the free-trial group were allowed 30 days of free Klorfasil use before purchase decision. Households who purchased Klorfasil were then followed up for 30, 60, and 180 days. At the last follow-up, respondents were asked if they would like to repurchase Klorfasil. Questionnaire survey and water quality assessment by residual-free chlorine were conducted in each survey. Chi-square test, $t$-test, and logistic regression were applied. The first purchase rate of the cost-sharing group was significantly higher than that of the free-trial group (79.2\% versus $67.3 \%)$. By contrast, the repurchase rate of the free-trial group was higher (82.9\% versus $66.3 \%)$. However, the overall repurchase rate was $71.6 \%$ and the proportion of long-term users was significantly higher in the cost-sharing group (56\% versus $47 \%$ ). Water treatment rates in the cost-sharing group were significantly higher than those in the free-trial group in the first and final surveys (odds ratio $[O R]=0.15, O R=0.32$ ). Households with high and medium economic status both had significantly higher purchase rates than low economic status households $(\mathrm{OR}=4.40$, $\mathrm{OR}=1.94)$. Households with higher educated respondents had significantly better water treatment practices $(O R=2.15)$. The free-trial approach did not increase the first purchase rate but increased the repurchase rate later. The cost-sharing approach significantly encouraged long-term usage.
\end{abstract}

\section{INTRODUCTION}

Access to clean water and sanitation plays a critical role in preventing diarrheal diseases in developing countries, especially in rural areas. ${ }^{1}$ Because piped water may not be feasible for households living in rural areas, various types of household water treatment and safe storage (HWTS) products have been developed and field-tested to effectively reduce diarrheal diseases around the world from $20 \%$ to $70 \% .^{1-16}$ Although considerable progress has been made, adoption and sustained usage of HWTS still remain low. ${ }^{6,15}$

Traditionally, HWTS products were distributed for free or at highly subsidized rates (cost-sharing) in developing countries, but which approach was more effective remains a highly controversial and complex issue in the global health arena. ${ }^{17-19}$ The rationale of free distribution was to maximize the likelihood that the population would receive the HWTS products to optimize its public health benefits. Proponents of free distribution asserted that "charging people for basic health care...[is] unfair" and that fees ensure that goods only reach "the richest of the poor."20,21

One study in Kenya reported that localized, short-lived freedistribution programs disproportionately benefit healthier women who can more easily travel to the distribution sites. ${ }^{22}$ In addition, study in Bangladesh reported that households who had received a free trial of one of four HWTS products did not use the products on most days. ${ }^{23}$ Moreover, the sustainability of such an approach remained an issue because of the lack of sustainable financial flow.

Conversely, the WHO and the world-renown economist, Jeffrey Sachs, have strongly advocated that subsidies are

* Address correspondence to Feng-Jen Tsai, Taipei Medical University, No. 250 Wu-Hsing Street, Taipei city 110, Taiwan. E-mail: jeanfjtsai@tmu.edu.tw appropriate to promote the use of goods that have positive externalities, such as health externalities from reductions in infectious disease. ${ }^{24}$ Advocates of cost-sharing further counter that "when products are given away free, the recipient often does not value them or even use them."18,25,26 However, according to researchers, there is evidence suggesting that increasing the price of the product by a marginal amount severely diminishes the number of users using the product. ${ }^{2,17,23}$ And, many nongovernmental organizations and program managers feel compelled to charge a fee to the user to screen out those who would not use the product for its intended use and recover some of the costs. ${ }^{27}$

Previous studies comparing the effectiveness of freedistribution and cost-sharing approaches showed diverse outcomes. $^{17-19}$ To add to the complexity, studies in Haiti, Guatemala, and Kenya have demonstrated how difficult it is to transition households from free water treatment products to purchases. ${ }^{9,16,28}$ Based on previous arguments and findings, researchers agree that social marketing strategies should be further explored to increase adoption rates of HWTS products. ${ }^{18,27,29}$

By definition, social marketing is "the application of proven concepts and techniques drawn from the commercial sector to promote changes in diverse socially important behaviors." 30 This approach is widely used in public health intervention with positive results. ${ }^{31,32}$ Studies on HWTS also recommend further research on using such approaches in developing countries. ${ }^{33}$

The free-trial method is a well-established commercial marketing technique that holds great potential for distributing chlorine disinfectants because it enables consumers to try an unfamiliar product without financial risk. ${ }^{29,34}$ To increase the willingness to pay and consistent treatment of water, it is plausible that people need to experiment with a new product before valuing it enough to purchase it. ${ }^{18,26,35}$ In addition, this approach might combine the benefit of both free-distribution 
approach and cost-sharing approach that it freely distribute the product in the beginning but charge with highly subsidized rates later. However, there are too few studies to ascertain whether or not this approach would be equally or more effective than previous conventional distribution strategies when targeting the poor in developing countries. ${ }^{26,27,29}$

To further understand the effectiveness of the free-trial approach, we conducted a cluster randomized control trial in Haiti. Haiti has the lowest rates of access to safe drinking water and sanitation in the Western Hemisphere. $6,16,36$ In 2015 , it was estimated that less than $50 \%$ of rural households have access to an improved source of drinking water, compared with $88 \%$ in urban areas. ${ }^{37}$ Although water quality and safety are emerging issues in the country, we conducted the study in rural Haiti with the hypothesis that the households in the free-trial group would be more likely to purchase HWTS products after their trial period and that they would also be more likely to treat their water continuously.

\section{MATERIALS AND METHODS}

Study design and procedure. Our study was conducted in seven rural villages (Barank, Dalegran, Bok Banik, Bouloume, Nan Kwa, Palmis Bas, and Savan Plat) in the Thomassique region of Central Plateau of Haiti from May to December 2016. These villages were chosen because of the similar distance from Medical Mission and their socioeconomic backgrounds. We partnered with Medical Missionaries, a nonprofit organization that has provided medical care and promoted public health programs in the Thomassique region in Haiti since 1998 to conduct this study. A cluster, randomized control trial design was applied to minimize contamination between the free-trial (intervention) and cost-sharing (control) group households and reduce selection bias.

The community health workers employed at Medical Missionaries were first trained to be surveyors of the study. The surveyors were trained in standard interviewing, data collection, and water testing techniques. A pilot test was conducted on seven households from each of the seven villages $(n=49)$, and the survey was revised accordingly.

Villages were then randomly allocated into free-trial (intervention) and cost-sharing (control) groups by lottery using sealed envelopes under the understanding of potential limitation of cluster approach as possible difference in household characteristics. The target sample size was calculated to be 381 based on the Raosoft sample size calculator. However, we aimed for at least 500 households because of the concern of possible attrition loss. Following the recommendations in the CDC's guide for conducting household surveys for water safety plans, surveyors were instructed to go door to door to find eligible candidates until a total of 80 households in each village were reached to gain a total of 480 households. ${ }^{7}$ In the end, 503 households were recruited and 497 enrolled in the study (Figure 1).

For all households, the surveyors administered a standardized baseline survey by questionnaire regarding HWTS after brief explanation and gaining written consent. ${ }^{14}$ The surveyors also provided the information regarding the benefits of using HWTS products and demonstrated how to use the product. In the free-trial group, heads of households were asked if they would like to experiment with the product for free for 30 days before making their purchase decision. In the control group, heads of households were asked if they would like to purchase the product for $20 \mathrm{HTG}$ (0.33 USD). The HWTS
Baseline

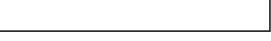

st follow up

( 30 days after baseline)

nd follow up

( 60 days after baseline)

rd follow up

(180 days after baseline)
503 household were recruited for this study 6 households declined to participate

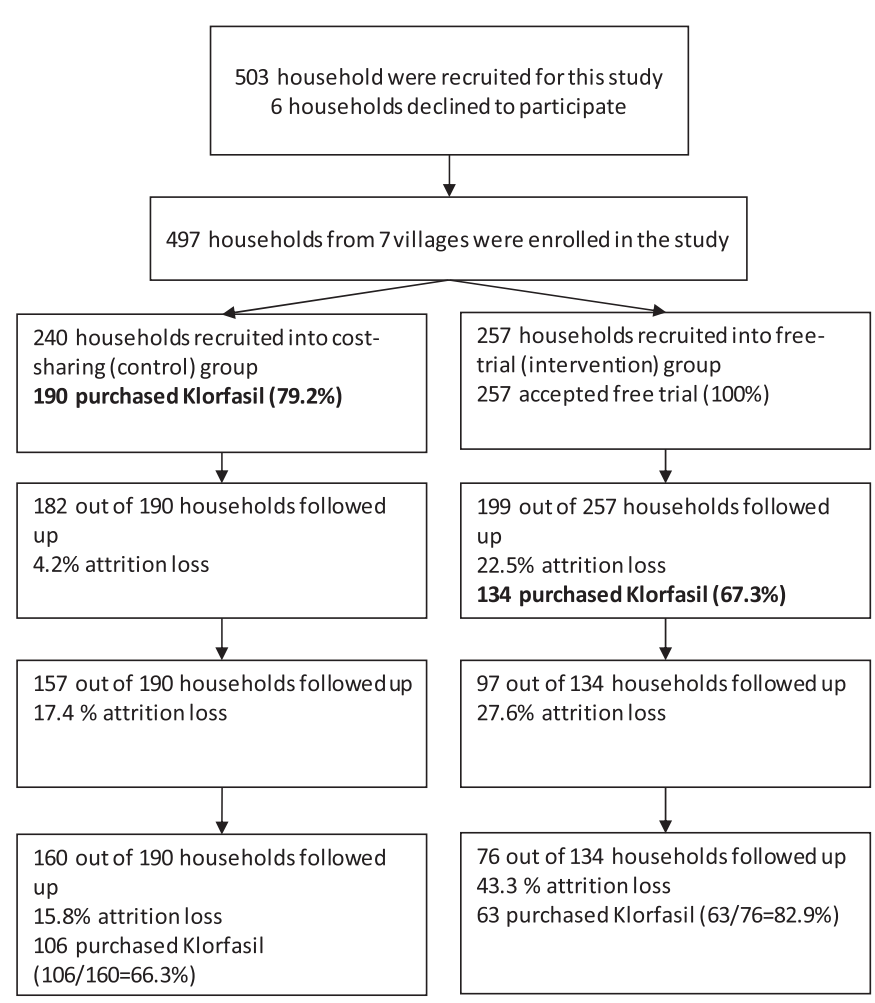

FigURE 1. Flowchart of participants and purchase decisions. 
product we used in the study is Klorfasil, a granulated chlorine-based HWTS product. ${ }^{38}$

After 30 days of baseline survey, free-trial group households were revisited and heads of households were asked if they would like to purchase the Klorfasil. Household water quality was also tested in the free-trial and control groups as a proxy to understand their water treatment behavior. Households in the control group who purchased the product were also revisited for monitoring their water quality, which represented their water treatment behavior. Then, households who purchased Klorfasil in both groups were further followed up for 60 and 180 days after the baseline survey for accessing their water quality. The surveyors revisited the participant a maximum of three times if the person was unavailable. At the final follow-up, participants were asked if they would like to continue purchasing Klorfasil.

Ethical consideration. The proposal for this study was approved by the Taipei Medical University Joint Institutional Review Board Commission (reference No: N201603084). And, this clinical trial is registered with the number as ISRCTN11717387 (https://doi.org/10.1186/ISRCTN11717387). Before conducting the study, oral consent was obtained from the leaders of the participating villages.

Participants. The inclusion criteria included heads of the households who were at least 18 years old and who had never used Klorfasil or any other type of long-term HWTS products before, with the exception of Aquatabs. ${ }^{39}$ Aquatabs are a one-time-use chlorine tablets commonly distributed during emergencies or during the rainy season, and do not qualify as long-term HWTS products.

Survey questionnaire. The survey questionnaire was adapted from the Joint WHO/The United Nations Children's Fund (UNICEF) tool kit. The standardized baseline survey questionnaires contained 40 questions including four sections: socioeconomic status and demographics of households and heads of households, knowledge and practices of water quality and safety including information regarding water collection frequency and time taken to collect water (6 items), belief of heads of households' toward water quality and safety (6 items), and hygiene practices including HWTS product usage (17 items). The survey was translated into Haitian Creole and back-translated into English for translation accuracy.

For the socioeconomic status and demographics, information regarding households' type of walls, roof, floor, and electricity were collected. Information on individual characteristics of the heads of households including gender, age, and educational level was also collected for analysis.

For knowledge and practices, information regarding water collection frequency and time taken to collect water was collected by questions such as "where do you usually get your water from?" and "How long does it take to go to your water source, collect water, and come back, each time (excluding time it takes to wait to collect water)?"

For belief toward water quality and safety, heads of households were asked about their belief toward water quality regarding current water source, such as "Do you think you can get sick from water?" and "Do you think your main source of water is safe to drink?"

Questions regarding hygiene practice evaluated participants' HWTS product usage to see if they could properly use the product. Information on the frequency of water collection was also collected. We also tested the household storage water for residual-free chlorine (RFC) levels, an objective measurement for water quality as a proxy for water treatment behavior.

Water quality assessment. Surveyors tested the household's storage water for RFC levels, which indicates that the water is free of microorganisms with a rapid chlorine test tablet (TesTabs) ${ }^{\star}$ on each subsequent visit. In accordance with the WHO's drinking-water standard, water with RFC levels $0.5 \mathrm{mg} / \mathrm{L}$ and greater was indicated as treated water in our study. ${ }^{14}$

Economic index. For households' economic status, we used the information including material type of the household (Table 1), latrine access, type of water source, and electricity status to represent their economic status because of the fact that those characteristics represented households' capacity of consumption. We gave scores to those characteristics. The more valuable the material, the higher the score we gave. The value of the materials was judged based on the local price of the materials. The improved source of water referred to water source as protected spring/public tap, bottled water, public standpipe, and so on. Further of the score calculations are described in Supplemental Appendix 1. The sum of the scores of economic status ranged from 3 to 9 . After taking the sum of economic indicators, we constructed a box and whiskers plot to set the cutoff points for "high," "medium," and "low" categories using the interquartile range as the average.

Data analysis. Three approaches were used in the study. Chi-squared tests were used to evaluate the differences of households' characteristics including household materials, type of water source, latrine access and economic status, and individual characteristics of heads of households including gender, age, education, and their belief toward water sanitation between the free-trial group and cost-sharing group. Independent $t$-test was used to evaluate the differences in water collection frequency and duration between groups. Multivariate regression models were run to evaluate the associations between households' HWTS product purchasing and water treatment behaviors and different approaches while controlling for households' economic status, demographic characteristics of heads of households, and their beliefs toward water safety and sanitation. Data were analyzed with SAS (version 9.4; SAS Institute Inc., Cary, NC) Odds ratios (ORs) and adjusted odds ratios (AOR) with $95 \% \mathrm{Cls}$ were calculated.

\section{RESULTS}

Participants' flow. Information of participants in each survey is shown in Figure 1. Through randomized arrangement, there were 240 households in the cost-sharing (control) group and 257 households in the free-trial (intervention) group that participated in baseline survey. In the free-trial group, there were 199 households in the first follow-up, 97 households in the second follow-up, and 76 households in the final follow-up that participated in the study. The overall attrition rate in following up with only households who bought Klorfasil is around $43.3 \%$. For the cost-sharing group, there were 182 households in the first follow-up (30 days after baseline survey), 157 households in the second follow-up (60 days after baseline survey), and 160 households in the final follow-up (180 days after baseline survey) that participated in the study. The overall attrition rate in the cost-sharing group was $15.8 \%$. 
TABLE 1

Comparison of household demographics between groups at baseline survey

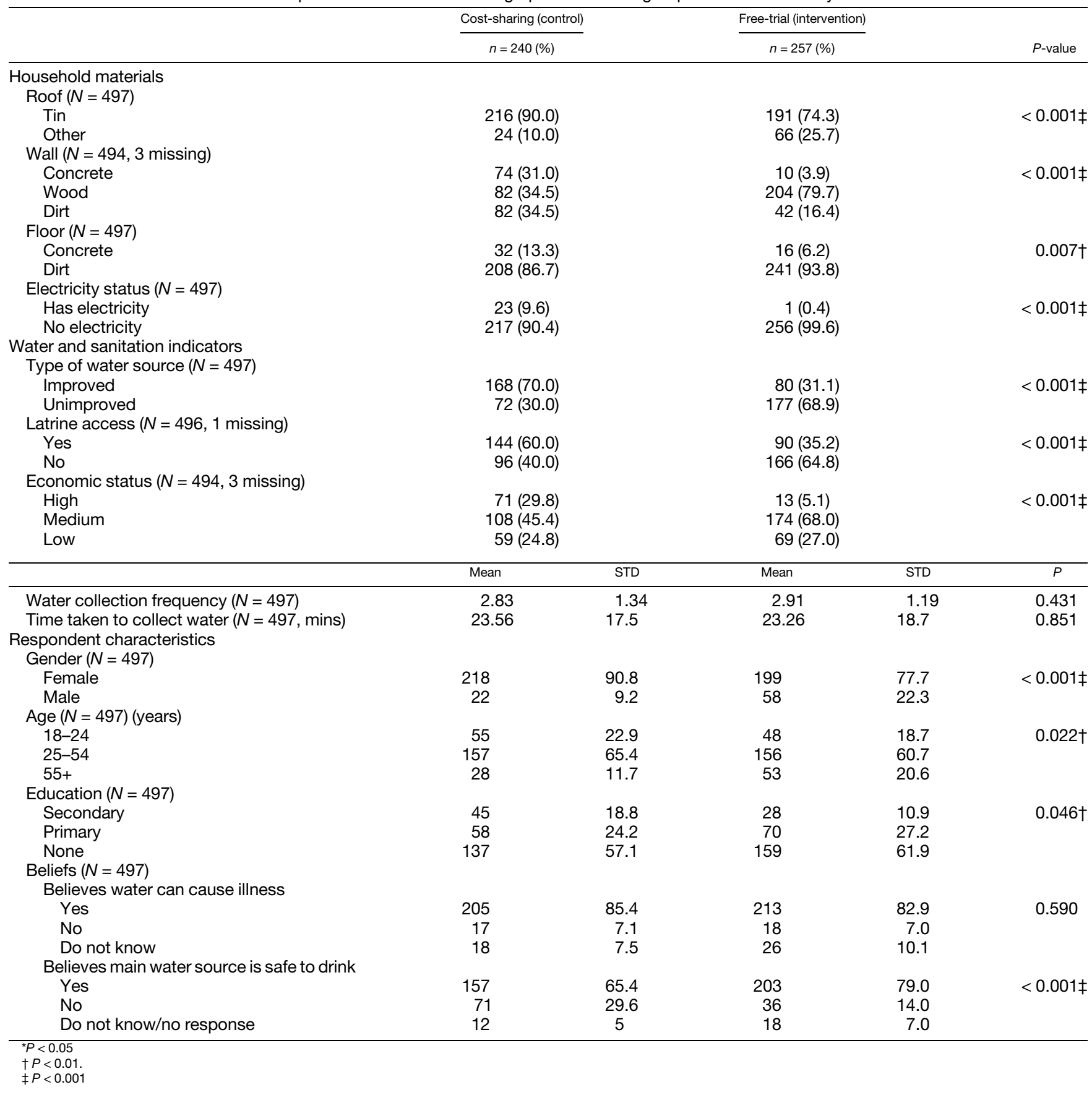

Klorfasil purchase behavior. At baseline, 190 of 240 (79.2\%) households in the control group purchased Klorfasil, whereas all 257 households accepted the free trial of Klorfasil. After the free trial period (30 days after baseline), 134 of 199 $(67.3 \%)$ households in the intervention group purchased Klorfasil. The purchase rate of households in the control group was significantly higher than that of those in the intervention group.

For the repurchase behavior among households who purchased Klorfasil before, there were 106 of the original 190 households in the cost-sharing group who continued to purchase Klorfasil at the third follow-up (after 180 days), whereas there were 63 of the original 134 households in the free-trial group who repurchased the Klorfasil. By using followed-up households in each survey as the denominator, the repurchase rate of households in the free-trial group was $82.9 \%$ (63/ $76)$, whereas the repurchase rate of the cost-sharing group was $66.3 \%$ (106/160). Different with the previous purchase behavior, the repurchase rate of the free-trial group was significantly higher than that of the control group.

Long-term sustained usage behavior. Under the goals of long-term sustained usage of Klorfasil, all participants were under the same condition of purchasing Klorfasil with a subsidized price after their first purchase. The overall repurchase 
rate of participants was $71.6 \%([106+63] /[160+76])$. There were $56 \%$ (106/190) final purchases with the first purchase in the cost-sharing group, whereas there were only $47 \%(63 / 134)$ final purchases in the free-trial group. The proportion of longterm users was significantly higher in the cost-sharing group than in the free-trial group.

Household demographics. Most households in both groups had tin roofs, although this proportion was significantly higher in the control group than in the intervention group (90.0\% versus $74.3 \%$, Table 1$)$. In the control group, there was an equal distribution of households having walls made of concrete (31.0\%), wood (34.5\%), and dirt (34.5\%). In comparison, a vast majority of households in the intervention group had walls made of wood $(79.7 \%)$. More than $85 \%$ of households in both groups had dirt floors and did not have electricity. More than twice as many households in the control group had access to an improved water source than those in the intervention group $(70.0 \%$ versus $31.1 \%)$. Almost twice as many households in the control group had a latrine in their household than those in the intervention group $(60.0 \%$ versus $35.2 \%$ ).

For the overall economic status, most of the households in the free-trial group were in the "medium" group (68\%). In the cost-sharing group, 29.8\% were in the "high" category, $45.4 \%$ were in the "medium" category, and $24.8 \%$ were in the "very low" category. All the household materials and economic status of households in the cost-sharing group were significantly better than those of households in the free-trial group.

Respondent characteristics. Most respondents in both groups were female, although this proportion was higher in the control group than in the intervention group $(90.8 \%$ versus $77.7 \%$, Table 1). In both groups, more than $60 \%$ of respondents were $25-54$ years old. Respondents in the control group had significantly better education level than respondents in the intervention group. More than $80 \%$ of heads of households in both groups believed that water could cause illness, but significantly more respondents in the intervention group believed that their main water source was safe to drink, even though only $35.2 \%$ of the intervention group had access to an improved source of water. For water collection frequency and time taken to collect water, there were no significant differences between groups.

Determinants of purchase and repurchase behavior. Determinants of purchase and repurchase behaviors via logistic regression analysis are reported in Table 2 . For the first purchase, univariate analysis suggested that households in the cost-sharing group were more likely to purchase Klorfasil. Although households in the cost-sharing group had significantly higher likelihood of purchasing Klorfasil in the univariate regression analysis, this was not significant in the multivariate regression model. After controlling for potential confounders, households with a high economic status had significantly higher purchase rates than households with a low economic status $(O R=4.40)$. Households with a medium economic status also purchased significantly more than households with a very low economic status $(O R=1.94)$.

For the repurchasing behavior, households in the free-trial group repurchased more than households in the cost-sharing group, although the difference was not significant. Households with respondents who finished secondary education were 6.25 times more likely to repurchase than households with respondents without formal education. The association between respondents' education level and repurchasing behavior was significant.

Water treatment results (water quality). Figure 2 showed the percentage of households with treated water by using the RFC level as an indicator. In both groups, water quality was highest in the beginning (from the first follow-up to the second follow-up) and dramatically decreased over time. At each follow-up, a greater proportion of households in the costsharing group treated their water when compared with households in the free-trial group. In the first follow-up and final follow-up, water treatment rates of the cost-sharing group was significantly higher than water treatment rates of

TABLE 2

Determinants of first purchase and repurchase at the final follow-up

\begin{tabular}{|c|c|c|c|c|c|c|}
\hline & \multicolumn{6}{|c|}{ Purchase behavior } \\
\hline & \multicolumn{3}{|c|}{ First purchase } & \multicolumn{3}{|c|}{ Repurchase } \\
\hline & AOR & $95 \% \mathrm{Cl}$ & $P$-value & AOR & $95 \% \mathrm{Cl}$ & $P$-value \\
\hline \multicolumn{7}{|l|}{ Distribution method } \\
\hline Intervention (free-trial) & 0.69 & $(0.434-1.100)$ & 0.119 & 1.75 & $(0.704-3.810)$ & 0.159 \\
\hline Control (cost-sharing) & - & - & - & - & - & - \\
\hline \multicolumn{7}{|c|}{ Socioeconomic characteristics } \\
\hline Gender & - & - & - & - & - & - \\
\hline Female & 1.28 & $(0.701-2.340)$ & 0.420 & 1.21 & $(0.427-3.452)$ & 0.717 \\
\hline Male & - & - & - & - & - & - \\
\hline \multicolumn{7}{|l|}{ Age (years) } \\
\hline $18-24$ & 1.22 & $(0.569-2.623)$ & 0.607 & 0.27 & $(0.070-1.018)$ & $0.053^{*}$ \\
\hline $25-54$ & 1.68 & $(0.928-3.043)$ & $0.087^{\star}$ & 1.69 & $(0.693-4.116)$ & 0.249 \\
\hline $55+$ & - & - & - & - & - & - \\
\hline \multicolumn{7}{|l|}{ Education } \\
\hline Secondary & 0.98 & $(0.453-2.097)$ & 0.948 & 6.25 & $(1.560-25.068)$ & $0.010 \dagger$ \\
\hline Primary & 1.00 & (0.574-1.736) & 0.995 & 1.53 & $(0.746-4.766)$ & 0.180 \\
\hline None & - & - & - & - & - & - \\
\hline \multicolumn{7}{|l|}{ Economic status } \\
\hline High & 4.40 & $(1.899-10.203)$ & $<0.001 \ddagger$ & 1.13 & $(0.406-3.134)$ & 0.816 \\
\hline Medium & 1.94 & $(1.172-3.203)$ & $0.010 \dagger$ & 1.53 & $(0.666-3.506)$ & 0.317 \\
\hline Low & - & - & - & - & - & - \\
\hline
\end{tabular}




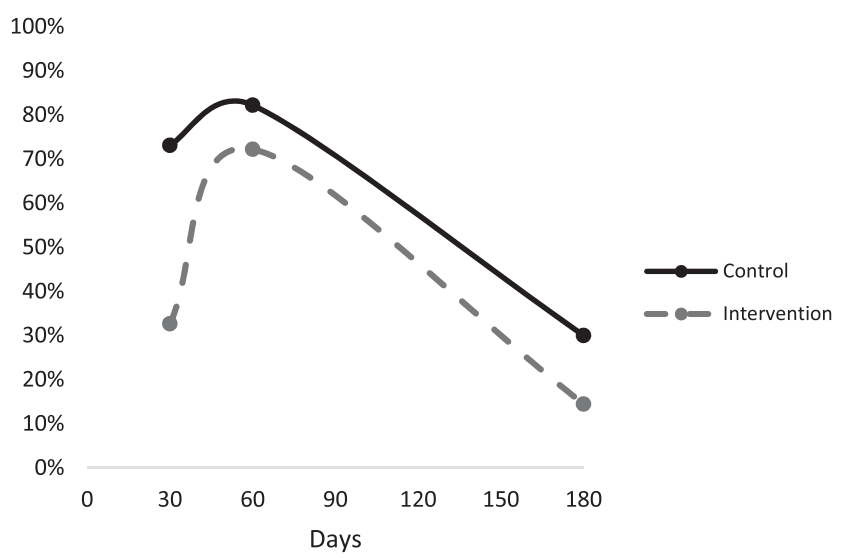

FIGURE 2. Households with positive residual-free chlorine levels (treated over a 180-day period).

the free-trial group. In the cost-sharing group, the percentage of households with positive RFC levels was $73.1 \%$ in the first follow-up, then increased to $82.2 \%$ in the second follow-up, and then decreased to $30 \%$ in the final follow-up. In the freetrial group, the percentage of households with positive RFC levels was $32.7 \%$ in the first follow-up, then sharply increased to $72.2 \%$ in the second follow-up, and then decreased to $14.5 \%$ in the final follow-up.

Determinants of water treatment at the first follow-up, second follow-up, and final follow-up. Determinants of water treatment at each survey are shown in Table 3. For the first follow-up, households in the free-trial group showed significantly less water treatment than households in the costsharing group $(\mathrm{OR}=0.15)$. And households with a high economic status had significantly less water treatment than households with a very low economic status $(\mathrm{OR}=0.47)$.

For the second follow-up, belief of heads of households toward water quality and safety was the only significant factor influencing water treatment behaviors. Households with respondents who believed the safety of their main water source had significantly less water treatment than those who did not believe in the safety of main water source $(\mathrm{OR}=0.31)$.

For the final follow-up, households in the free-trial group showed significantly less water treatment than households in the cost-sharing group. And, households where respondents had primary education $(\mathrm{OR}=2.22)$ had significantly better water treatment than households with no official education.

\section{DISCUSSION}

The purpose of our study was to investigate whether or not the free-trial approach would increase purchase and sustained usage rates of the Klorfasil chlorine dispenser. The strength of our study was that it took real-life purchase decisions and objective measurements of water quality in comparison with willingness-to-pay studies or other artificial experiments evaluating purchase and water treatment behavior. ${ }^{40}$ Our study design evaluated whether or not households actually treated their water by not informing them our arrival date and time to avoid households' strong tendency to give social desirable answers. However, although we intended to include villages with similar socioeconomic conditions, our

TABLE 3

Determinants of water treatment outcomes (free chlorine level) at each survey

\begin{tabular}{|c|c|c|c|c|c|c|c|c|c|}
\hline & \multicolumn{9}{|c|}{ Water treatment behavior } \\
\hline & \multicolumn{3}{|c|}{ First follow-up } & \multicolumn{3}{|c|}{ Second follow-up } & \multicolumn{3}{|c|}{ Final follow-up } \\
\hline & AOR & $95 \% \mathrm{Cl}$ & $P$-value & AOR & $95 \% \mathrm{Cl}$ & $P$-value & AOR & $95 \% \mathrm{Cl}$ & $P$-value \\
\hline \multicolumn{10}{|l|}{ Distribution method } \\
\hline Intervention (free-trial) & 0.15 & $(0.091-0.259)$ & $<0.001 \ddagger$ & 0.74 & $(0.369-1.483)$ & 0.395 & 0.45 & $(0.194-1.024)$ & $0.006 \dagger$ \\
\hline Control (cost-sharing) & - & - & - & - & - & - & - & - & - \\
\hline \multicolumn{10}{|c|}{ Socioeconomic characteristics } \\
\hline \multicolumn{10}{|c|}{ Gender } \\
\hline Female & 0.76 & $(0.390-1.481)$ & 0.420 & 0.72 & $(0.237-2.198)$ & 0.567 & 3.02 & $(0.784-11.668)$ & 0.108 \\
\hline Male & - & - & - & - & - & - & - & - & - \\
\hline \multicolumn{10}{|l|}{ Age (years) } \\
\hline $18-24$ & 1.75 & $(0.723-4.236)$ & 0.214 & 2.45 & $(0.601-10.006)$ & 0.211 & 0.69 & $(0.192-2.483)$ & 0.571 \\
\hline $25-54$ & 1.32 & $(0.656-2.649)$ & 0.438 & 1.15 & $(0.435-3.047)$ & 0.778 & 0.49 & $(0.183-1.304)$ & 0.153 \\
\hline $55+$ & - & - & - & - & - & - & - & - & - \\
\hline \multicolumn{10}{|l|}{ Education } \\
\hline Secondary & 0.93 & $(0.435-1.969)$ & 0.841 & 2.57 & $(0.621-10.604)$ & 0.193 & 1.41 & $(0.493-4.021)$ & 0.523 \\
\hline Primary & 0.81 & $(0.465-1.415)$ & 0.460 & 1.02 & $(0.469-2.209)$ & 0.965 & 2.22 & $(0.996-4.925)$ & $0.051^{\star}$ \\
\hline None & - & - & - & - & - & - & - & - & - \\
\hline \multicolumn{10}{|l|}{ Economic status } \\
\hline High & 0.47 & $(0.212-1.039)$ & $0.062^{*}$ & 1.07 & $(0.362-3.141)$ & 0.907 & 2.44 & $(0.773-7.691)$ & 0.128 \\
\hline Medium & 0.71 & $(0.396-1.273)$ & 0.250 & 0.88 & $(0.387-2.003)$ & 0.762 & 1.77 & $(0.633-4.943)$ & 0.277 \\
\hline Low & - & - & - & - & - & - & - & - & - \\
\hline \multicolumn{10}{|c|}{ Beliefs } \\
\hline \multicolumn{10}{|c|}{ Believes water can cause illness } \\
\hline Yes & - & - & - & - & - & - & - & - & - \\
\hline No & - & - & - & - & - & - & - & - & - \\
\hline \multicolumn{10}{|c|}{$\begin{array}{l}\text { Believes main water source is } \\
\text { safe to drink }\end{array}$} \\
\hline Yes & 0.75 & $(0.429-1.300)$ & 0.302 & 0.31 & $(0.123-0.794)$ & $0.015 \dagger$ & 1.09 & $(0.530-2.233)$ & 0.819 \\
\hline No & - & - & - & - & - & - & - & - & - \\
\hline
\end{tabular}

$+P<0.01$

$\neq P<0.001$ 
households in cost-sharing group had significantly better socioeconomic status than households in free-trial group.

We hypothesized that experimentation with the product may help convince users to purchase a chlorine dispenser and make their water safe to drink while reducing waste of resources. Contrary to our expectations, the free-trial did not increase purchase rates for Klorfasil at first time. From our multivariate regression models, it seems clear that economic status was the main determinant of whether the respondent purchased Klorfasil, even after controlling for confounding variables. Our findings contradict Berry et al.'s study, who asserted that there was no conclusive evidence supporting any significant association between wealth indicators and willingness to pay for cleaner water. ${ }^{41}$ However, at the final follow-up, respondents in the free-trial group were more likely to repurchase Klorfasil than the cost-sharing group (82.9\% [63/76] versus $66.2 \%$ [106/160]). Our findings also suggested that heads of households with higher educational levels were more likely to repurchase the product at the final follow-up. Although it is unclear why more respondents in the free-trial group chose to repurchase Klorfasil after 180 days had passed, there may be a connection in improving purchasing behavior in the long run. As Dupas et al., ${ }^{38}$ have suggested, there could be other nonmonetary costs that we have not accounted for that may have attributed to their water treatment behavior.

Although the purchase rate of the free-trial group did not increase as we expected, our study result showed the high percentage of repurchase rates among all participants who first purchased the product. Also, our study result showed significantly higher percentage of long-term users of Klorfasil in the cost-sharing group. As indicated in previous studies, the cost-sharing approach provided subsides to reduce the burden of using HWTS for participants, our findings support that subsidies may encourage long-term purchase and usage of HWTS products. ${ }^{18}$ Our study supports further adaptation of the cost-sharing approach in the long run.

As for water treatment practices, our findings confirm that respondents in the free-trial group were less likely to treat their water, even after controlling for important socioeconomic factors. During the free-trial period, less than half of the households in the free-trial group treated their water. But the gap between groups diminished at the second follow-up visit, in which all participants had purchased Klorfasil. This finding might have partially supported the argument that people who have paid for the product would value and use the product. However, at the final follow-up, the number of households who treated their water at the time of visit was dramatically reduced, although they all bought the product. Only $30 \%$ of households of the cost-sharing group had treated water, and only $14.5 \%$ households in the free-trial group treated water at the final follow-up. This finding suggests that people who bought the product would use it frequently in the beginning, but the usage rate would diminish over time. Also, it is unclear why respondents in the free-trial group were less likely to treat water even after paying for the product. There is a further need to understand how to sustain consistent HWTS product usage over time.

Importantly, our study showed the effects of education in repurchasing and water treatment behavior. Our results indicated that households with a better economic status were more likely to purchase the HWTS product in the beginning, but households with respondents with at least elementary levels of education were more likely to repurchase the HWTS product and to continuously treat water. Our findings show that respondents who believed that their main water source was safe to drink were less likely to treat their water further supports the importance of focusing on education for sustaining water treatment practices, which aligns with Ahuja's Kenyan study. ${ }^{2}$

Our research study had some limitations. Despite randomizing villages to the free-trial or cost-sharing group, there were many significant differences in household characteristics. Another limitation was that there may be other variables responsible for purchase and water treatment decisions that we were not able to obtain. Last, the sample size was significantly reduced because of attrition loss at the final follow-up, especially in the free-trial group. Although we attempted to control for confounders in the regression models, our statistical power was reduced by attrition loss.

\section{CONCLUSION}

In conclusion, our findings showed that the free-trial approach did not incentivize new users to purchase Klorfasil, but over time, participants in the free-trial group continued to purchase Klorfasil at the final follow-up. The overall repurchase rate of participants was as high as $71.6 \%$. In addition, our results reported a significantly higher percentage of long-term users of Klorfasil in the cost-sharing group. One's household economic status played a major role in determining the purchasing behavior, but the educational level of the head of household was also a significant factor that associated with households' HWTS repurchasing behavior. In addition, our research affirms that water treatment practices diminish over time, and approaches to reduce the nonmonetary costs associated with water treatment practices should be explored. Based on our study's findings, we suggest that further research is needed to explore other viable social marketing approaches to increase purchase and sustain water treatment practices.

Received August 15, 2019. Accepted for publication November 13, 2019.

Published online January 20, 2020.

Note: Supplemental Appendix appears at www.ajtmh.org.

Acknowledgments: We thank Medical Missionaries Global Health Fellowship program for hosting M. W. in Haiti. We also thank them for their support in facilitating the study process. We thank Frederique Jean-Baptiste for her contribution in translating and back translating the surveys in Haitian Kreyòl and reviewing the study design. We thank Jon Steele, founder of Klorfasil, for the donation of 500 chlorine dispensers used in the study. We thank Bernard Celestin for his contribution in overseeing the supply chain and other logistics behind procuring and transporting the Klorfasil chlorine dispensers for the study. We thank the Medical Missionaries for their offer of a Global Health Fellowship for M. W.'s stay in Haiti.

Authors' addresses: Michael Wu, Centers for Disease Control and Prevention, Atlanta, GA, E-mail: michael.j.wu316@gmail.com. FengJen Tsai, Master Program in Global Health and Development, College of Public Health, Taipei Medical University, Taipei City, Taiwan, E-mail: jeanfjtsai@tmu.edu.tw. Chia-Ping Lin, Master Program in Global Health and Development, School of Public Health, Taipei City, Taiwan, E-mail: spadeh2294@tmu.edu.tw.

\section{REFERENCES}

1. Fiebelkorn AP, Person B, Quick RE, Vindigni SM, Jhung M, Bowen A, Riley PL, 2012. Systematic review of behavior change research on point-of-use water treatment interventions in countries 
categorized as low-to medium-development on the human development index. Soc Sci Med 75: 622-633.

2. Ahuja A, Kremer M, Zwane AP, 2010. Providing safe water: evidence from randomized evaluations. Annu Rev Resour Econ 2: 237-256.

3. Centers for Disease Control and Prevention, 2013. Safe Water System-Chlorination, USA.

4. Clasen TF, Alexander KT, Sinclair D, Boisson S, Peletz R, Chang $\mathrm{HH}$, Majorin F, Cairncross S, 2015. Interventions to improve water quality for preventing diarrhoea. Cochrane Database Syst Rev CD004794.

5. Fewtrell L, Kaufmann RB, Kay D, Enanoria W, Haller L, Colford JM, 2005. Water, sanitation, and hygiene interventions to reduce diarrhoea in less developed countries: a systematic review and meta-analysis. Lancet Infect Dis 5: 42-52.

6. Harshfield E, Lantagne D, Turbes A, Null C, 2012. Evaluating the sustained health impact of household chlorination of drinking water in rural Haiti. Am J Trop Med Hyg 87: 786-795.

7. Lantagne DS, Gallo W, 2008. Safe Water for the Community: a Guide for Establishing a Community-Based Safe Water System Program. Atlanta, GA: Centers for Disease Control and Prevention.

8. Lantagne DS, Quick R, Mintz ED, 2006. Household water treatment and safe storage options in developing countries: a review of current implementation practices. Wilson Quarterly, Woodrow Wilson International Center for Scholars. Washington, DC: Environmental Change and Security Program.

9. Luby SP, Mendoza C, Keswick BH, Chiller TM, Hoekstra RM, 2008. Difficulties in bringing point-of-use water treatment to scale in rural Guatemala. Am J Trop Med Hyg 78: 382-387.

10. Sobsey MD, Stauber CE, Casanova LM, Brown JM, Elliott MA, 2008. Point of use household drinking water filtration: a practical, effective solution for providing sustained access to safe drinking water in the developing world. Environ Sci Technol 42: 4261-4267.

11. The World Bank, 2014. Clean Water, Improved Sanitation, Better Health. Availible at: https://www.worldbank.org/en/events/ 2014/09/30/haiti-clean-water-improved-sanitation-betterhealth. Accessed August 1, 2019.

12. WHO/UNICEF, 2014. Joint Monitoring Programme for Water Supply and Sanitation. Progress on Drinking Water and Sanitation: 2014 Update. Geneva, Switzerland: World Health Organization.

13. World Health Organization, 2009. Scaling up Household Water Treatment Among Low-Income Populations. Geneva, Switzerland: WHO.

14. WHO/UNICEF, 2012. A Toolkit for Monitoring and Evaluating Household Water Treatment and Safe Storage Programmes. Geneva, Switzerland: World Health Organization.

15. Rosa G, Clasen T, 2010. Estimating the scope of household water treatment in low- and medium-income countries. Am J Trop Med Hyg 82: 289-300.

16. Lantagne D, Clasen T, 2013. Effective use of household water treatment and safe storage in response to the 2010 Haiti earthquake. Am J Trop Med Hyg 89: 426-433.

17. Ashraf N, Berry J, Shapiro JM, 2010. Can higher prices stimulate product use? Evidence from a field experiment in Zambia. Am Econ Rev 100: 2383-2413.

18. Cohen J, Dupas P, 2010. Free distribution or cost-sharing? Evidence from a randomized malaria prevention experiment. Q J Econ 125: $1-45$.

19. Kremer M, Holla A, 2008. Pricing and Access: Lessons from Randomized Evaluation in Education and Health. Washington, DC: Center for Global Development Working Paper No. 158.

20. Benn H, 2006. Meeting Our Promises: Basic Services for Everyone, Everywhere. London, United Kingdom: White Paper speech by the Secretary of State for International Development, 16 February.
21. McNeil Jr. DG, 2005. A Program to Fight Malaria in Africa Draws Questions. New York Times.

22. Kremer M, Miguel E, Mullainathan S, 2014. Source Dispensers and Home Delivery of Chlorine in Kenya. Innovations for Poverty Action. Available at: https://www.povertyactionlab.org/evaluation/ source-dispensers-and-home-delivery-chlorine-kenya. Accessed July 30, 2019.

23. Luoto J, Mahmud M, Albert J, Luby S, Najnin N, Unicomb L, Levine DI, 2012. Learning to dislike safe water products: results from a randomized controlled trial of the effects of direct and peer experience on willingness to pay. Environ Sci Technol 46: 6244-6251.

24. Easterly W, Easterly WR, 2006. The White Man's Burden: Why the West's Efforts to Aid the Rest Have Done So Much III and So Little Good. New York, NY: Penguin Press, 436.

25. Holla A, Kremer M, 2009. Pricing and Access: Lessons from Randomized Evaluations in Education and Health. Washington, DC: Center for Global Development Working Paper No. 158.

26. Dupas $P, 2014$. Short-run subsidies and long-run adoption of new health products: evidence from a field experiment. Econometrica 82: 197-228.

27. Null C, Kremer M, Miguel E, Hombrados JG, Meeks R, Zwane AP, 2012. Willingness to Pay for Cleaner Water in Less Developed Countries: Systematic Review of Experimental Evidence. London, United Kingdom: The International Initiative for Impact Evaluation (3iE)

28. Kremer M, Null C, Miguel E, Zwane AP, 2008. Trickle Down: Diffusion of Chlorine for Drinking Water Treatment in Kenya. UC-Berkeley, Working Paper.

29. Evans WD, Pattanayak SK, Young S, Buszin J, Rai S, Bihm JW, 2014. Social marketing of water and sanitation products: a systematic review of peer-reviewed literature. Soc Sci Med 110: $18-25$.

30. Andreasen AR, 2002. Marketing social marketing in the social change marketplace. J Public Pol Market 21: 3-15.

31. Evans WD, 2006. How social marketing works in health care. BMJ 332: $1207-1210$.

32. Smith WA, 2006. Social marketing: an overview of approach and effects. Inj Prev 12: i38-i43.

33. USAID, 2007. Best Practices in Social Marketing Safe Water SoIution for Household Water Treatment: Lessons Learned from Population Services International Field Programs. Washington, DC: USAID.

34. Wood S, Foster J, Kols A, 2012. Understanding why women adopt and sustain home water treatment: insights from the Malawi antenatal care program. Soc Sci Med 75: 634-642.

35. Dupas $P, 2009$. What matters (and what does not) in households' decision to invest in malaria prevention? Am Econ Rev 99: 224-230.

36. Etienne CF, Tappero JW, Marston BJ, Frieden TR, Kenyon TA, Andrus JK, 2013. Cholera elimination in Hispaniola. Am J Trop Med Hyg 89: 615-616.

37. UNICEF, 2014. At a glance: Haiti. Available at: http://www.unicef. org/infobycountry/haiti_2014.html. Accessed August 1, 2019.

38. Klorfasil, 2012. Klorfasil Safe Water Program. Available at: www. klorfasil.org. Accessed August 1, 2019.

39. Aquatabs, 2019. Safer Water, Safer World. Available at: http:// www.aquatabs.com/home/. Accessed August 1, 2019.

40. Burt Z, Njee RM, Mbatia Y, Msimbe V, Brown J, Clasen TF, Malebo HM, Ray I, 2017. User preferences and willingness to pay for safe drinking water: experimental evidence from rural Tanzania. Soc Sci Med 173: 63-71.

41. Berry J, Fischer G, Guiteras RP, 2015. Eliciting and utilizing willingness to pay: evidence from field trials in northern Ghana. CEPR Discussion Paper No. DP10703. Available at: https:// ssrn.com/abstract=2630151. 